\title{
Pharmacotherapy for chronic hemorrhagic radiation proctitis
}

\begin{abstract}
Endoscopic thermal therapy and formalin are being increasingly recommended for the treatment of chronic hemorrhagic radiation proctitis. It may be too early, however, to discard pharmacologic agents from the management process, especially in medical institutions where specialized equipment is unavailable. We prospectively assessed the effectiveness of medical therapy in 14 consecutive patients with chronic hemorrhagic radiation proctitis from July 1999 to June 2001. All 14 subjects were women (mean age 56 years), 13 of whom had had radio-therapy for Cancer of the cervix. The median time to onset of Symptoms following irradiation was 16 months. Six patients had a hemoglobin level of $<8 \mathrm{~g} / \mathrm{dl}$, and blood transfusion was required in 11 patients. In five patients (36\%) initially treated with hydrocortisone enemas prior to referral, this treatment continued; and the remaining nine patients were commenced on sucralfate enemas. Two patients given rectal hydrocortisone continued to bleed and were treated with sucralfate enemas and topical formalin, respectively. Rectal sucralfate Suspension effectively procured symptomatic alleviation in all 11 patients. Rectal bleeding recurred in two patients who had been managed exclusively with hydrocortisone and sucralfate enemas, respectively, over a mean follow-up of 6 months. Both patients were managed with topical formalin, which con-trolled their Symptoms. Even though the number of subjects in this study is small, sucralfate enema can be recommended as an effective first-line agent for managing patients with chronic hemorrhagic radiation proctitis. The use of more specialized therapy can therefore be reserved for cases where primary treatment failure occurs with sucralfate therapy.
\end{abstract}

Keyword: Chronic hemorrhagic radiation proctitis; Radiotherapy; Cervix cancer 